\title{
An axiomatization of the nucleolus of assignment markets
}

\author{
Francesc Llerena · Marina Núñez · Carles \\ Rafels
}

Received: date / Accepted: date

\begin{abstract}
On the domain of two-sided assignment markets with agents' reservation values, the nucleolus is axiomatized as the unique solution that satisfies consistency with respect to Owen's reduced game and symmetry of maximum complaints of the two sides. As an adjunt, we obtain a geometric characterization of the nucleolus by means of a strong form of the bisection property that characterizes the intersection between the core and the kernel of a coalitional game in Maschler et al. (1979).
\end{abstract}

Keywords cooperative games $\cdot$ assignment game $\cdot$ core $\cdot$ nucleolus

\section{Introduction}

The assignment game is a coalitional game that represents a two-sided market situation. In this market there exist a finite set of sellers, each one with an indivisible object on sell, and a finite set of buyers willing to buy at most one object each. Each agent has a reservation value that is what he or she obtains if not matched with an agent on the opposite side. Every buyer-seller pair $(i, j)$ is attached to a real number $a_{i j}$ that represents the value that this pair can attain if matched together. From these valuations, we obtain the assignment matrix $A$. The worth of each coalition is the total profit that can be obtained by optimally matching buyers and sellers in the coali-

The authors acknowledge the support from research grants ECO2011-22765 (Ministerio de Ciencia e Innovación and FEDER), 2009SGR900 and 2009SGR960 (Generalitat de Catalunya). We are also grateful to an Associated Editor and anonymous referees for their helpful comments.

Francesc Llerena

Dep. de Gestió d'Empreses, Universitat Rovira i Virgili-CREIP, Av. de la Universitat, 1, 43204 Reus, Spain

E-mail: francesc.llerena@urv.cat

Marina Núñez $\cdot$ Carles Rafels

Dep. de Matemàtica Econòmica, Financera i Actuarial, Universitat de Barcelona, Av. Diagonal, 690, 08034

Barcelona, Spain

E-mail: mnunez@ub.edu,E-mail: crafels@ub.edu 
tion. When reservation values are null and the assignment matrix is non-negative, our game is the one introduced by Shapley and Shubik (1972).

Coalitional game theory analyzes how the agents can share the profit of an optimal pairing, taking into account the worth of all possible coalitions. The most studied solution concept in this model has been the core, the set of efficient allocations that are coalitionally rational. Shapley and Shubik prove that the core of the assignment game is non-empty and it can be described just in terms of the assignment matrix, with no need of the associated characteristic function.

Other solutions have been considered for the assignment game: Thompson's fair division point (Thompson, 1981), the kernel or symmetrically pairwise bargained allocations (Rochford 1984), the nucleolus (Solymosi and Raghavan 1994), the Shapley value (Hoffmann and Sudhölter, 2007; van den Brink and Pintér, 2012) and the von Neumann-Morgenstern stable sets (Núñez and Rafels 2013). However, axiomatic characterizations of solutions in this framework have been mainly focused on the core (Sasaki, 1995; Toda, 2003 and 2005).

On the general class of coalitional games, the prenucleolus (that for the assignment game coincides with the nucleolus) has been axiomatized by Sobolev (1975) by means of covariance, anonimity and the reduced game property of Davis and Maschler (1965). Potters (1991) also characterizes the nucleolus on the class of balanced games ${ }^{1}$ by means of the above reduced game property. However, both aforementioned sets of axioms do not characterize the nucleolus on the class of assignment games since, for instance, the Davis and Maschler reduced game of an assignment game needs not remain inside this class. Moreover, it seems desirable an axiomatization of the nucleolus of the assignment game in terms of axioms that are not stated by means of the characteristic function but by means of the data of the assignment market.

In the present paper, on the domain of assignment games with agents' reservation values, the nucleolus is uniquely determined by only two axioms: consistency with respect to Owen's reduced game and symmetry of maximum complaints of the two sides. Consistency is based on the derived game introduced by Owen (1992). Roughly speaking, symmetry of maximum complaints of the two sides only requires that at each solution outcome of a market with as many buyers as sellers, the most dissatisfied buyer has the same complaint as the most dissatisfied seller, where we interpret the dissatisfaction of an agent with a given outcome as the difference between his reservation value and the amount that this outcome allocates to him.

As a by-product of the axiomatization of the nucleolus, we obtain a geometric characterization of the nucleolus. Maschler et al. (1979) provide a geometrical characterization for the intersection of the kernel and the core of a coalitional game, showing that those allocations that lie in both sets are always the midpoint of certain bargaining range between each pair of players. In the case of the assignment game, this means that the kernel can be determined as those core allocations where the maximum amount that can be transferred, without getting outside the core, from one agent to his/her optimally matched partner equals the maximum amount that he/she can receive from this partner, also remaining inside the core (Rochford 1984; Driessen

\footnotetext{
1 In fact Potters characterizes the nucleolus in a more general class of games.
} 
1999). We now state that the nucleolus of the assignment game can be characterized by requiring this bisection property be satisfied not only for optimally matched pairs but also for optimally matched coalitions.

Preliminaries on assignment games are in Section 2. Section 3 explores the property of consistency with respect to Owen's reduced game. In Section 4 we prove the axiomatic characterization of the nucleolus and deduce a geometric characterization.

\section{Preliminaries}

Let $U$ and $U^{\prime}$ be two countable disjoint sets, the first one formed by all potential buyers and the second one formed by all potential sellers. An assignment market with agents' reservation values, hereafter an assignment market, is a quintuple $\gamma=$ $\left(M, M^{\prime}, A, p, q\right)$. The sets $M \subset U$ and $M^{\prime} \subset U^{\prime}$ are two finite sets of buyers and sellers respectively (the two sides of the market) of cardinality $|M|=m$ and $\left|M^{\prime}\right|=m^{\prime}$, with $M \cup M^{\prime}$ non-empty. Matrix $A=\left(a_{i j}\right)_{(i, j) \in M \times M^{\prime}}$ is such that for all $(i, j) \in M \times M^{\prime}$, the real number $a_{i j}$ denotes the worth obtained by the pair $(i, j)$ if they trade. Finally, $p \in \mathbb{R}^{M}$ and $q \in \mathbb{R}^{M^{\prime}}$ where, for all $i \in M, p_{i}$ is the reservation value of buyer $i$ if she remains unpaired with any seller (and similarly for $q_{j}$ for all $j \in M^{\prime}$ ). Notice that neither $a_{i j}$ nor $p_{i}$ or $q_{j}$ are constrained to be non-negative. When the market has null reservation prices we will just describe it as $\left(M, M^{\prime}, A\right)$.

A matching $\mu$ between $S \subseteq M$ and $T \subseteq M^{\prime}$ is a bijection from a subset of $S$ to a subset of $T$. We denote by $\operatorname{Dom}(\mu) \subseteq S$ and $\operatorname{Im}(\mu) \subseteq T$ the corresponding domain and image. If $i \in S$ and $j \in T$ are related by $\mu$ we indistinctly write $(i, j) \in \mu, j=\mu(i)$ or $i=\mu^{-1}(j)$. We denote by $\mathscr{M}(S, T)$ the set of matchings between $S$ and $T$. Given an assignment market $\gamma=\left(M, M^{\prime}, A, p, q\right)$, for all $S \subseteq M, T \subseteq M^{\prime}$ and $\mu \in \mathscr{M}(S, T)$ we write

$$
v(S, T ; \mu)=\sum_{(i, j) \in \mu} a_{i j}+\sum_{i \in S \backslash \operatorname{Dom}(\mu)} p_{i}+\sum_{j \in T \backslash \operatorname{Im}(\mu)} q_{j},
$$

with the convention that any summation over an empty set of indices is zero.

A matching $\mu \in \mathscr{M}\left(M, M^{\prime}\right)$ is optimal for the assignment market $\gamma=\left(M, M^{\prime}, A, p, q\right)$ if for all $\mu^{\prime} \in \mathscr{M}\left(M, M^{\prime}\right)$ it holds $v\left(M, M^{\prime} ; \mu\right) \geq v\left(M, M^{\prime} ; \mu^{\prime}\right)$. The set of optimal matchings for the assignment market $\gamma$ is denoted by $\mathscr{M}_{\gamma}^{*}\left(M, M^{\prime}\right)$.

With any assignment market $\gamma=\left(M, M^{\prime}, A, p, q\right)$, we associate a game in coalitional form $\left(M \cup M^{\prime}, w_{\gamma}\right)$ (assignment game) with player set $M \cup M^{\prime}$ and characteristic function $w_{\gamma}$ defined as follows: for all $S \subseteq M$ and $T \subseteq M^{\prime}$,

$$
w_{\gamma}(S \cup T)=\max \{v(S, T ; \mu) \mid \mu \in \mathscr{M}(S, T)\} .
$$

Notice that by (1) and (2) we have that $w_{\gamma}(\{i\})=p_{i}$ for all $i \in M$ and $w_{\gamma}(\{j\})=$ $q_{j}$ for all $j \in M^{\prime}$. This assignment game, that allows for agents' reservation values, is a generalization of the assignment game of Shapley and Shubik (1972) (that is, an assignment game with non-negative matrix and null reservation values) and was introduced by Owen (1992) and also used by Toda (2003, 2005).

We denote by $\Gamma_{\mathrm{AG}}$ the set of all assignment markets $\gamma=\left(M, M^{\prime}, A, p, q\right)$, and also, for simplicity of notation, the set of their corresponding assignment games. Since we 
will deal with consistency properties, we allow for the emptiness of one side of the market. ${ }^{2}$ The set of assignment games $\Gamma_{\mathrm{AG}}$ is closed by strategic equivalence. In fact, it can be shown that every assignment game in $\Gamma_{A G}$ is strategically equivalent to an assignment game in the sense of Shapley and Shubik. ${ }^{3}$ As a consequence, Shapley and Shubik's results on the core of the assignment game extend to $\Gamma_{\mathrm{AG}}$.

Given an assignment market $\gamma=\left(M, M^{\prime}, A, p, q\right)$, a payoff vector is $z=(u, v) \in$ $\mathbb{R}^{M} \times \mathbb{R}^{M^{\prime}}$ where $u_{i}$ stands for the payoff to buyer $i \in M$ and $v_{j}$ stands for the payoff to seller $j \in M^{\prime}$. We write $z_{\mid S}$ or $\left(u_{\mid S \cap M}, v_{\mid S \cap M^{\prime}}\right)$ to denote the projection of a payoff vector $z$ to agents in coalition $S \subseteq M \cup M^{\prime}$. Also, $z(S)=\sum_{i \in S} z_{i}$, with $z(\emptyset)=0$. An imputation of $\gamma$ is a payoff vector $(u, v)$ that is efficient, $u(M)+v\left(M^{\prime}\right)=w_{\gamma}\left(M \cup M^{\prime}\right)$, and individually rational, $u_{i} \geq p_{i}$ for all $i \in M$ and $v_{j} \geq q_{j}$ for all $j \in M^{\prime}$. We denote by $I(\gamma)$ the set of imputations of the assignment market $\gamma$.

The core of the assignment market is always non-empty and it is formed by those efficient payoff vectors $(u, v) \in \mathbb{R}^{M} \times \mathbb{R}^{M^{\prime}}$ that satisfy coalitional rationality for mixed-pair coalitions and one-player coalitions:

$$
C(\gamma)=\left\{\begin{array}{l|l}
(u, v) \in \mathbb{R}^{M} \times \mathbb{R}^{M^{\prime}} & \begin{array}{l}
\sum_{i \in M} u_{i}+\sum_{j \in M^{\prime}} v_{j}=w_{\gamma}\left(M \cup M^{\prime}\right), \\
u_{i}+v_{j} \geq a_{i j} \text { for all }(i, j) \in M \times M^{\prime}, \\
u_{i} \geq p_{i} \text { for all } i \in M, v_{j} \geq q_{j} \text { for all } j \in M^{\prime}
\end{array}
\end{array}\right\} .
$$

Moreover, if $\mu$ is an optimal matching of $\gamma$, any core allocation $(u, v) \in \mathbb{R}^{M} \times \mathbb{R}^{M^{\prime}}$ satisfies

$$
\begin{gathered}
u_{i}+v_{j}=a_{i j} \text { for all }(i, j) \in \mu, \\
u_{i}=p_{i} \text { for all } i \in M \backslash \operatorname{Dom}(\mu), \\
v_{j}=q_{j} \text { for all } j \in M^{\prime} \backslash \operatorname{Im}(\mu) .
\end{gathered}
$$

One single-valued core selection of the assignment market is the nucleolus. This solution, that was introduced for arbitrary coalitional games by Schmeidler (1969), only relies on the worth of individual coalitions and mixed-pair coalitions when applied to the assignment game. Given an assignment market $\gamma=\left(M, M^{\prime}, A, p, q\right)$, with any imputation $(u, v) \in I(\gamma)$ we associate a vector $\theta(u, v)$ whose components are $a_{i j}-u_{i}-v_{j}$, for all $(i, j) \in M \times M^{\prime}, p_{i}-u_{i}$ for all $i \in M$ and $q_{j}-v_{j}$ for all $j \in M^{\prime}$, non-increasingly ordered. Then, the nucleolus of the assignment market $\gamma$ is the imputation $\eta(\gamma)$ that minimizes $\theta(u, v)$ with respect to the lexicographic order over the set of imputations: $\theta(\eta(\gamma)) \leq_{\text {Lex }} \theta(u, v)$ for all $(u, v) \in I(\gamma)$. This means that, for all $(u, v) \in I(\gamma)$, either $\theta(\eta(v))=\theta(u, v)$ or $\theta(\eta(v))_{1}<\theta(u, v)_{1}$ or there exists $k \in\left\{2, \ldots, m m^{\prime}+m+m^{\prime}\right\}$ such that $\theta(\eta(v))_{i}=\theta(u, v)_{i}$ for all $1 \leq i \leq k-1$ and $\theta(\eta(v))_{k}<\theta(u, v)_{k}$.

\footnotetext{
2 If $\gamma$ is an assignment market with $M^{\prime}=\emptyset$, then it is easy to see that the associated assignment game $\left(M, w_{\gamma}\right)$ given by (2) is the modular game generated by the vector of reservation values $p \in \mathbb{R}^{M}$, that is, $w_{\gamma}(S)=\sum_{i \in S} p_{i}$, for all $S \subseteq M$. Similarly, if $\gamma=\left(M, M^{\prime}, A, p, q\right)$ with $M=\emptyset$, then $w_{\gamma}(T)=\sum_{j \in T} q_{j}$, for all $T \subseteq M^{\prime}$.

3 Two games $(N, v)$ and $(N, w)$ are strategically equivalent if there exist $\alpha>0$ and $d \in \mathbb{R}^{N}$ such that $w(S)=\alpha v(S)+\sum_{i \in S} d_{i}$. Let $\gamma=\left(M, M^{\prime}, A, p, q\right)$ be an assignment market where $A=\left(a_{i j}\right)_{(i, j) \in M \times M^{\prime}}, p \in$ $\mathbb{R}^{M}, q \in \mathbb{R}^{M^{\prime}}$, and let $\tilde{\gamma}=\left(M, M^{\prime}, \tilde{A}\right)$ be an assignment market with null reservation values and matrix $\tilde{A}=\left(\tilde{a}_{i j}\right)_{(i, j) \in M \times M^{\prime}}$ given by $\tilde{a}_{i j}:=\max \left\{0, a_{i j}-p_{i}-q_{j}\right\}$, for all $(i, j) \in M \times M^{\prime}$. Then, as the reader can easily check, $w_{\gamma}(S \cup T)=w_{\tilde{\gamma}}(S \cup T)+\sum_{i \in S} p_{i}+\sum_{j \in T} q_{j}$, for all $S \subseteq M$ and $T \subseteq M^{\prime}$.
} 


\section{Consistency with respect to Owen's reduced game and the core of the assigment game}

In this section we consider a consistency property, with respect to a certain reduction of the market, that will be satisfied not only by the core but also by the nucleolus. We begin by introducing the concept of a solution on the domain of assignment markets. The next two definitions follow Toda (2005).

Definition 1 Let $\gamma=\left(M, M^{\prime}, A, p, q\right) \in \Gamma_{\mathrm{AG}}$. A payoff vector $(u, v) \in \mathbb{R}^{M} \times \mathbb{R}^{M^{\prime}}$ is feasible-by-matching if there exists $\mu \in \mathscr{M}\left(M, M^{\prime}\right)$ such that

(i) $u_{i}=p_{i}$ for all $i \in M \backslash \operatorname{Dom}(\mu), v_{j}=q_{j}$ for all $j \in M^{\prime} \backslash \operatorname{Im}(\mu)$, and

(ii) $u_{i}+v_{j}=a_{i j}$ for all $(i, j) \in \mu$.

In the above definition, the payoff vector $(u, v)$ and the matching $\mu$ are said to be compatible with each other. Notice that a matching that is compatible with a feasible-by-matching payoff vector need not be an optimal matching. This notion of feasibility-by-matching, being quite restrictive, emphasizes the bilateral nature of the market. Note also that any core allocation of the assignment market is trivially feasible-by-matching, since it is compatible with any optimal matching.

Definition 2 A solution on $\Gamma_{\mathrm{AG}}$ is a correspondence $\sigma$ that associates a non-empty subset of feasible-by-matching payoff vectors with each $\gamma \in \Gamma_{\mathrm{AG}}$.

Note that the core correspondence and the mapping that gives to each player his/her reservation value (compatible with the empty matching) are examples of solutions on the class of assignment markets.

If $\gamma=\left(M, M^{\prime}, A, p, q\right) \in \Gamma_{\mathrm{AG}}$, we write $\sigma(\gamma)$ to denote the image of this assignment market by a given solution $\sigma$.

Consistency is a standard property used to analyze the behavior of solutions with respect to reduction of population. Roughly speaking, a solution is consistent if whenever we reduce the game to a subset of agents and the excluded agents are paid according to a solution payoff, the projection of this payoff to the remaining agents still belongs to the solution of the reduced game. Different consistency ${ }^{4}$ notions depend on the different definitions for the reduced game, that is, the different ways in which the remaining agents can reevaluate their coalitional capabilities. Probably, the best known notion of consistency is based on Davis and Maschler reduced game (Davis and Maschler, 1965). Peleg (1986) uses the above consistency notion to characterize the core on the domain of all coalitional games. However, it turns out that the Davis and Machler reduced game of an assignment game may not be an assignment game (see Owen, 1992). To overcome this drawback, Owen introduces the following reduced market.

Definition 3 Let $\gamma=\left(M, M^{\prime}, A, p, q\right)$ be an assignment market, $\emptyset \neq T \subseteq M \cup M^{\prime}$, and $z=(u, v) \in \mathbb{R}^{M} \times \mathbb{R}^{M^{\prime}}$. The Owen's reduced assignment market relative to $T$ at $z$ is

\footnotetext{
4 For comprehensive surveys on the consistency principles, the reader is referred to Driessen (1991) and Thomson (2003).
} 
$\gamma^{T, z}=\left(T \cap M, T \cap M^{\prime}, A^{T}, p^{T, z}, q^{T, z}\right)$, where $A^{T}=A_{\mid(T \cap M) \times\left(T \cap M^{\prime}\right)}$ and

$$
\begin{aligned}
& p_{i}^{T, z}=\max \left\{p_{i}, \max _{j \in M^{\prime} \backslash T}\left\{a_{i j}-v_{j}\right\}\right\}, \text { for all } i \in T \cap M, \\
& q_{j}^{T, z}=\max \left\{q_{j}, \max _{i \in M \backslash T}\left\{a_{i j}-u_{i}\right\}\right\}, \text { for all } j \in T \cap M^{\prime} .
\end{aligned}
$$

The Owen's reduced assignment game relative to $T$ at $z$ is the coalitional game associated to the Owen's reduced assignment market $\gamma^{T, z}$, that is $\left(T, w_{\gamma^{T, z}}\right)$.

The interpretation of Owen's reduced market is as follows. Once agents not in $T$ have left the market taking their corresponding payoff in $z$, the agents in $T$ interact in the submarket defined by the submatrix $A^{T}=A_{\mid(T \cap M) \times\left(T \cap M^{\prime}\right)}$ but must reevaluate their reservation values, since the outside option has been modified. Each agent $i \in$ $T \cap M$ has the choice of remaining unmatched (thus getting the original reservation value) or matching somebody, say $j$, outside $T$, which leaves an income $a_{i j}-z_{j}$. The best of these choices determines the new reservation value of agent $i \in T \cap M$. Similarly, agents in $T \cap M^{\prime}$ reevaluate their reservation value.

Owen's reduction is closely related to the Davis and Maschler reduction when applied to an assignment game. Recall at this point the definition of the Davis-Maschler reduced game $\left(T, w_{D M}^{T, y}\right)$ relative to a game $(N, w)$, a coalition $T \subseteq N, T \neq \emptyset$, at a payoff vector $y \in \mathbb{R}^{N}$ by $w_{D M}^{T, y}(T)=w(N)-y(N \backslash T), w_{D M}^{T, y}(\emptyset)=0$ and

$$
w_{D M}^{T, y}(S)=\max \{w(S \cup R)-y(R) \mid \quad R \subseteq N \backslash T\}, \quad \text { for all } \quad S \subseteq T, S \neq T, S \neq \emptyset .
$$

In the setting of efficient payoff vectors $y$, the efficiency level in the Davis-Maschler reduced game relative to a coalition $T$ is exactly the total payoff to that coalition: $w_{D M}^{T, y}(T)=y(T)$.

Owen (1992) proved that for an assignment game $\gamma$, Owen's reduced game at a core allocation $z \in C(\gamma)$ is the superadditive cover of the Davis and Maschler reduced game at the same core allocation: ${ }^{5}$

$$
\left(T, w_{\gamma^{T, z}}\right)=\left(T, \widehat{\left(w_{\gamma}\right)_{D M}^{T, z}}\right) .
$$

Next we define consistency with respect to Owen's reduced game.

A solution $\sigma$ on $\Gamma_{\mathrm{AG}}$ satisfies

- consistency with respect to Owen's reduced game if for all $\gamma=\left(M, M^{\prime}, A, p, q\right) \in$ $\Gamma_{\mathrm{AG}}$, all $\emptyset \neq T \subseteq M \cup M^{\prime}$ and all $z \in \sigma(\gamma)$, it holds $z_{\mid T} \in \sigma\left(\gamma^{T, z}\right)$.

5 For properties regarding coalitional games, see Peleg and Sudhölter (2003). In particular, given a coalitional game $(N, v)$, the superadditive cover $(N, \hat{v})$ is the game with the following characteristic function: for all $S \subseteq N$,

$$
\hat{v}(S)=\max _{P \in \mathscr{P}_{S}} \sum_{T_{i} \in P} v\left(T_{i}\right),
$$

where $\mathscr{P}_{S}$ is the set of partitions of $S$. 
It is quite straightforward to see that the core of the assignment market satisfies consistency with respect to Owen's reduced game. Take $\emptyset \neq T \subseteq M \cup M^{\prime}$. Notice first that for all $z=(u, v) \in C(\gamma)$ we deduce $u_{i} \geq p_{i}^{T, z}$ for all $i \in M \cap T$ and $v_{j} \geq p_{j}^{T, z}$ for all $j \in M^{\prime} \cap T$, and hence $z(S) \geq w_{\gamma}, z(S)$ for all $S \subseteq T$. Now, taking this into account together with (8), we get

$$
z(T) \geq w_{\gamma, z}(T)=\widehat{\left(w_{\gamma}\right)_{D M}^{T, z}}(T) \geq\left(w_{\gamma}\right)_{D M}^{T, z}(T)=z(T)
$$

which results in $z(T)=w_{\gamma^{T, z}}(T)$.

As for the nucleolus, it is known from Miquel and Núñez (2011) that whenever a game and its superadditive cover have the same efficiency level, they have the same nucleolus. Hence, taking (8) into account, and also the fact that the nucleolus satisfies consistency with respect to Davis and Maschler reduced game on the domain of balanced games (Potters, 1991), we get that if $\eta=\eta\left(w_{\gamma}\right)$ and $\emptyset \neq T \subseteq M \cup M^{\prime}$,

$$
\eta\left(w_{\gamma}^{T, \eta}\right)=\eta\left(\left(w_{\gamma}\right)_{D M}^{T, \eta}\right)=\eta_{\mid T} .
$$

This completes the proof of the following proposition.

Proposition 1 On the domain of assignment markets $\Gamma_{A G}$, the core and the nucleolus satisfy consistency with respect to Owen's reduced game.

We prove in the next proposition that, on the domain $\Gamma_{A G}$, any solution $\sigma$ satisfying consistency with respect to Owen's reduced game selects a subset of the core, that is, $\sigma(\gamma) \subseteq C(\gamma)$ for all $\gamma \in \Gamma_{A G}$. This result is needed in the axiomatization theorem (Theorem 1), but it is also of interest on its own.

Proposition 2 On the domain of assignment markets $\Gamma_{A G}$, consistency with respect to Owen's reduced game implies core selection.

Proof Let $\sigma$ be a solution on $\Gamma_{\mathrm{AG}}$ satisfying consistency with respect to Owen's reduced game. Let $\gamma=\left(M, M^{\prime}, A, p, q\right)$ be an assignment market and $z=(u, v) \in \sigma(\gamma)$. If $M \neq \emptyset$ and $M^{\prime}=\emptyset$, then the only possible matching is $\mu=\emptyset$ and feasibility-bymatching of the solution implies $z=p$ and $C(\gamma)=\{z\}$. Similarly, if $M=\emptyset$ and $M^{\prime} \neq \emptyset$, then $z=q$ and $C(\gamma)=\{z\}$.

Assume now that $M \neq \emptyset$ and $M^{\prime} \neq \emptyset$. Consistency (with respect to Owen's reduced game) of $\sigma$ applied to singletons $T=\{i\}, T=\{j\}, i \in M, j \in M^{\prime}$, gives

$$
\begin{aligned}
& u_{i} \in \sigma\left(\gamma^{\{i\}, z}\right) \quad \text { whereas } \quad u_{i}=p_{i}^{\{i\}, z}=\max \left\{p_{i}, \quad \max _{j \in M^{\prime}}\left\{a_{i j}-v_{j}\right\}\right\}, \\
& v_{j} \in \sigma\left(\gamma^{\{j\}, z}\right) \quad \text { whereas } \quad v_{j}=q_{j}^{\{j\}, z}=\max \left\{q_{j}, \quad \max _{i \in M}\left\{a_{i j}-u_{i}\right\}\right\},
\end{aligned}
$$

since nobody is matched in one-person assignment games and also by feasibility-bymatching of solutions. Hence,

$$
u_{i} \geq p_{i}, \quad v_{j} \geq q_{j}, \quad \text { and } \quad u_{i}+v_{j} \geq a_{i j} \quad \text { for all } \quad i \in M, j \in M^{\prime} .
$$


In the remainder, let $\mu$ be an optimal matching of $\gamma=\left(M, M^{\prime}, A, p, q\right)$ and $\mu^{\prime}$ a matching that is compatible with $z=(u, v) \in \sigma(\gamma)$ (such a matching exists because of feasibility-by-matching of $z \in \sigma(\gamma)$ ). Using expression (10), it follows

$$
\begin{aligned}
w_{\gamma}\left(M \cup M^{\prime}\right) & =\sum_{i \in \operatorname{Dom}(\mu)} a_{i \mu(i)}+p(M \backslash \operatorname{Dom}(\mu))+q\left(M^{\prime} \backslash \operatorname{Im}(\mu)\right) \\
& \leq \sum_{i \in \operatorname{Dom}(\mu)}\left[u_{i}+v_{\mu(i)}\right]+u(M \backslash \operatorname{Dom}(\mu))+v\left(M^{\prime} \backslash \operatorname{Im}(\mu)\right)=u(M)+v\left(M^{\prime}\right) \\
& =\sum_{i \in \operatorname{Dom}\left(\mu^{\prime}\right)}\left[u_{i}+v_{\mu^{\prime}(i)}\right]+u\left(M \backslash \operatorname{Dom}\left(\mu^{\prime}\right)\right)+v\left(M^{\prime} \backslash \operatorname{Im}\left(\mu^{\prime}\right)\right) \\
& =\sum_{i \in \operatorname{Dom}\left(\mu^{\prime}\right)} a_{i \mu^{\prime}(i)}+p\left(M \backslash \operatorname{Dom}\left(\mu^{\prime}\right)\right)+q\left(M^{\prime} \backslash \operatorname{Im}\left(\mu^{\prime}\right)\right),
\end{aligned}
$$

where the last but one equality is due to a simple reordering of terms and the last one to the fact that $\mu^{\prime}$ is compatible with $z=(u, v)$. Because $\mu$ is an optimal matching of $\gamma$, the only inequality must be an equality and hence, the efficiency $z\left(M \cup M^{\prime}\right)=$ $u(M)+v\left(M^{\prime}\right)=w_{\gamma}\left(M \cup M^{\prime}\right)$ holds and the proof of $z=(u, v) \in C(\gamma)$ is completed.

\section{Axiomatic and geometric characterizations of the nucleolus}

In this section, we characterize axiomatically the nucleolus on the class of assignment games with agents' reservation values by means of two axioms, the first of them being consistency with respect to Owen's reduced game. Due to the bilateral structure of the market, we look for a second axiom that guarantees some balancedness between groups. As a by-product of the following axiomatization we will derive a geometric characterization that determines the position of the nucleolus inside the core.

Given an assignment market $\gamma=\left(M, M^{\prime}, A, p, q\right)$, if $j \in M^{\prime}$ and $(u, v)$ is a payoff vector, $q_{j}-v_{j}$ measures the difference between player's $j$ reservation value $q_{j}$ and the amount $v_{j}$ he has been paid. Thus, the higher this difference is, the more dissatisfied the agent is with the payoff vector. Sector $M^{\prime}$ as a whole can measure its degree of dissatisfaction by $\max _{j \in M^{\prime}}\left\{q_{j}-v_{j}\right\}$, and in this way we define the complaint of a sector as the maximum dissatisfaction of its agents. Analogously, the complaint of sector $M$ is $\max _{i \in M}\left\{p_{i}-u_{i}\right\}$.

A solution $\sigma$ on $\Gamma_{\mathrm{AG}}$ satisfies

- symmetry of maximum complaints of the two sides if for all square market, $\gamma=\left(M, M^{\prime}, A, p, q\right) \in \Gamma_{\mathrm{AG}}$ with $|M|=\left|M^{\prime}\right|$, and all $(u, v) \in \sigma(\gamma)$, it holds

$$
\max _{i \in M}\left\{p_{i}-u_{i}\right\}=\max _{j \in M^{\prime}}\left\{q_{j}-v_{j}\right\} .
$$

It is worth to remark that symmetry of maximum complaints of the two sides, although defined by means of excesses of individual coalitions, is far from the definition of the nucleolus since it does never compare these excesses across different imputations. 
Note that, when imposed on solution concepts that are core selections, the above property is not much demanding. Indeed, for those markets with as many buyers as sellers, imposing symmetry of maximum complaints of the two sides results on the selection of a hypersurface. For instance, in a square Shapley and Shubik's assignment game $\left(M, M^{\prime}, A\right)$, imposing symmetry of maximum complaints of the two sides is equivalent to imposing $\min _{i \in M}\left\{u_{i}\right\}=\min _{j \in M^{\prime}}\left\{v_{j}\right\}$ to the solution outcomes, and in general there are infinitely many core allocations that satisfy this equality. Figure 1 depicts the core of a $2 \times 2$-assignment game and the piece-wise linear curve $A-B-C-D$ formed by the subset of core allocations at which both sectors have the same complaint.

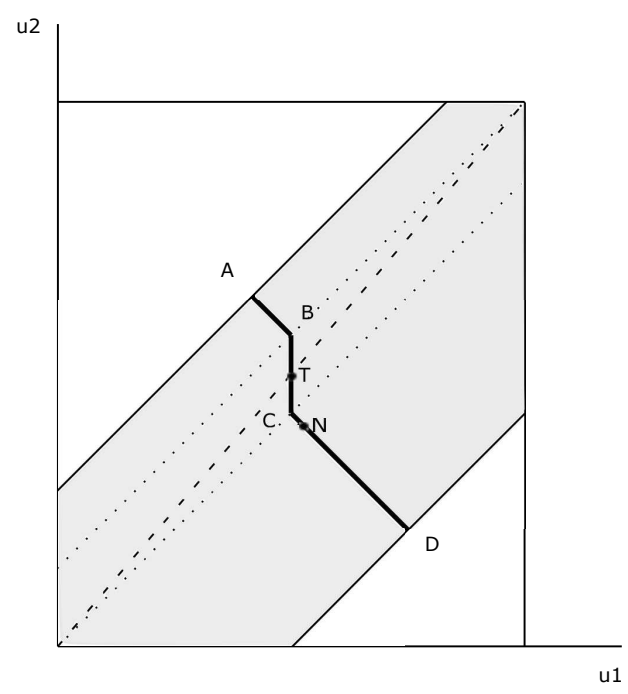

Fig. 1

A more formal geometric interpretation of symmetry of maximum complaints of the two sides will follow after the proof of Theorem 1. It is not difficult to realize that the midpoint between the buyers-optimal core allocation and the sellers-optimal core allocation (point $\mathrm{T}$ in the figure), that is known as Thompson's fair division point (Thompson 1981), satisfies symmetry of maximum complaint of the two sides. Next we prove that the nucleolus (denoted by $\mathrm{N}$ in Figure 1) also satisfies this symmetry property.

Proposition 3 On the domain of assignment markets $\Gamma_{A G}$, the nucleolus satisfies symmetry of maximum complaints of the two sides.

Proof Let $\gamma=\left(M, M^{\prime}, A, p, q\right) \in \Gamma_{A G}$ and let $\eta=\eta(\gamma)$ be the nucleolus of the assignment market $\gamma$ with $|M|=\left|M^{\prime}\right|$. Define the two real numbers $\varepsilon_{1}, \varepsilon_{2}$ by 


$$
\varepsilon_{1}=-\max _{i \in M}\left\{p_{i}-\eta_{i}\right\} \quad \text { and } \quad \varepsilon_{2}=-\max _{j \in M^{\prime}}\left\{q_{j}-\eta_{j}\right\} .
$$

Notice that $\varepsilon_{1} \geq 0$ and $\varepsilon_{2} \geq 0$ due to core-membership of $\eta$. We show $\varepsilon_{1}=\varepsilon_{2}$. Assume without loss of generality that $\varepsilon_{2} \geq \varepsilon_{1}$. Put $\delta=\varepsilon_{2}-\varepsilon_{1}$ and construct the payoff vector $z=(u, v) \in \mathbb{R}^{M} \times \mathbb{R}^{M^{\prime}}$ by $u_{i}=\eta_{i}+\frac{\delta}{2}$ for all $i \in M$ and $v_{j}=\eta_{j}-\frac{\delta}{2}$ for all $j \in M^{\prime}$. Clearly, $u_{i}+v_{j}=\eta_{i}+\eta_{j}$ for all $i \in M$ and $j \in M^{\prime}$. It is left to the reader to verify that $z \in C(\gamma)$ by checking $u_{i} \geq p_{i}$ for all $i \in M$ as well as $v_{j} \geq q_{j}$ for all $j \in M^{\prime}$.

Notice that if $C(\gamma)$ is a singleton, then trivially $\varepsilon_{1}=\varepsilon_{2}$.

If $C(\gamma)$ is not a singleton, assume $\varepsilon_{2}>\varepsilon_{1}$. Since for all $(i, j) \in M \times M^{\prime}$ it holds

$$
a_{i j}-u_{i}-v_{j}=a_{i j}-\eta_{i}-\eta_{j},
$$

to lexicographically compare the vector of ordered excesses of $z=(u, v)$ and $\eta$, we only need to consider the excesses of individual coalitions. ${ }^{6}$

We now write $N=M \cup M^{\prime}$ and prove $\max _{k \in N}\left\{w_{\gamma}(\{k\})-z_{k}\right\}=-\frac{\varepsilon_{1}+\varepsilon_{2}}{2}$. Indeed,

$$
\max _{i \in M}\left\{p_{i}-u_{i}\right\}=\max _{i \in M}\left\{p_{i}-\eta_{i}\right\}-\frac{\delta}{2}=-\varepsilon_{1}-\frac{\delta}{2}=-\frac{\varepsilon_{1}+\varepsilon_{2}}{2},
$$

as well as

$$
\max _{j \in M^{\prime}}\left\{q_{j}-v_{j}\right\}=\max _{j \in M^{\prime}}\left\{q_{j}-\eta_{j}\right\}+\frac{\delta}{2}=-\varepsilon_{2}+\frac{\delta}{2}=-\frac{\varepsilon_{1}+\varepsilon_{2}}{2} .
$$

From this, together with $\varepsilon_{2}>\varepsilon_{1}$ and (12), it follows that

$\max _{k \in N}\left\{w_{\gamma}(\{k\})-z_{k}\right\}=-\frac{\varepsilon_{1}+\varepsilon_{2}}{2}<-\varepsilon_{1}=\max \left\{-\varepsilon_{1}, \quad-\varepsilon_{2}\right\}=\max _{k \in N}\left\{w_{\gamma}(\{k\})-\eta_{k}\right\}$.

Since $z \in C(\gamma)$, the latter strict inequality is in contradiction with $\eta$ to represent the nucleolus. Hence, $\varepsilon_{1}=\varepsilon_{2}$ and this concludes the proof.

We are now ready to state and prove the axiomatic characterization of the nucleolus.

Theorem 1 On the domain of assignment markets $\Gamma_{A G}$, the only solution satisfying consistency with respect to Owen's reduced game and symmetry of maximum complaints of the two sides is the nucleolus.

Proof From Propositions 1 and 3 we know that the nucleolus satisfies both properties. To show uniqueness assume there exists a solution $\sigma$ on $\Gamma_{\mathrm{AG}}$ satisfying consistency with respect to Owen's reduced game and symmetry of maximum complaints of the two sides.

Let $\gamma=\left(M, M^{\prime}, A, p, q\right) \in \Gamma_{A G}$ and $z=(u, v) \in \sigma(\gamma)$. Let $\eta$ be the nucleolus of the market $\gamma$. We show $z=\eta$.

\footnotetext{
6 The following property is well known (see, for instance, Potters and Tijs, 1992). For any $n \in \mathbb{N}$ we define the map $\theta: \mathbb{R}^{n} \longrightarrow \mathbb{R}^{n}$ which arranges the coordinates of a point in $\mathbb{R}^{n}$ in non-increasing order. Take $x, y \in \mathbb{R}^{n}$ such that $\theta(x)$ is lexicographically not greater than $\theta(y)$. Take now any $z \in \mathbb{R}^{p}$ and consider the vectors $(x, z),(y, z) \in \mathbb{R}^{n+p}$. Then, $\theta(x, z)$ is lexicographically not greater than $\theta(y, z)$.
} 
Notice first that, from Proposition 2, $\sigma$ satisfies core selection and thus $z \in C(\gamma)$. Let $\mu \in \mathscr{M}_{\gamma}^{*}\left(M, M^{\prime}\right)$ be an optimal matching. If $\mu=\emptyset$, since both $z$ and $\eta$ belong to the core, we have $z=\eta$. Assume then that $\mu \neq \emptyset$ and $z \neq \eta$. For any $\emptyset \neq S \subseteq M$ such that $|S|=|\mu(S)|$ let us consider the Owen's reduced market relative to $T=S \cup \mu(S)$ at $z$. Notice that under the current assumptions, such a coalition $S$ always exists. Firstly, by consistency (with respect to Owen's reduced game) of $\sigma$, it holds $z_{\mid T} \in \sigma\left(\gamma^{T, z}\right)$. Secondly, since $|S|=|\mu(S)|$, by symmetry of maximum complaints of the two sides applied to $\sigma$ and the Owen's reduced assignment market $\gamma^{T, z}$ relative to $T$ at $z$, we conclude that

$$
\max _{i \in S}\left\{p_{i}^{T, z}-z_{i}\right\}=\max _{j \in \mu(S)}\left\{q_{j}^{T, z}-z_{j}\right\}
$$

Inserting (6) and (7) in the above equality, we obtain

$$
\begin{aligned}
& \max _{i \in S}\left\{\max \left\{p_{i}-z_{i}, \quad \max _{k \in M^{\prime} \backslash \mu(S)}\left\{a_{i k}-z_{k}-z_{i}\right\}\right\}\right\} \\
& =\max _{j \in \mu(S)}\left\{\max \left\{q_{j}-z_{j}, \quad \max _{k \in M \backslash S}\left\{a_{k j}-z_{k}-z_{j}\right\}\right\}\right\} .
\end{aligned}
$$

So far, it holds

$$
\max _{\substack{i \in S, k \in M^{\prime} \backslash \mu(S)}}\left\{p_{i}-z_{i}, \quad a_{i k}-z_{i}-z_{k}\right\}=\max _{\substack{j \in \mu(S), k \in M \backslash S}}\left\{q_{j}-z_{j}, \quad a_{k j}-z_{k}-z_{j}\right\}
$$

for all non-empty coalition $S \subseteq M$ with $|S|=|\mu(S)|$ and all $z \in \sigma(\gamma)$, being $\sigma$ a solution satisfying consistency with respect to Owen's reduced game and symmetry of maximum complaints of the two sides.

Since, by assumption, $z$ differs from the nucleolus $\eta$, without loss of generality we can assume there exists a coalition $S^{*} \subseteq M, S^{*} \neq \emptyset$, such that

$$
z_{i}>\eta_{i} \quad \text { for all } i \in S^{*} \quad \text { and } \quad z_{k} \leq \eta_{k} \quad \text { for all } k \in M \backslash S^{*} .
$$

Note that all agents in $S^{*}$ are matched by the given optimal matching $\mu$. Indeed, if there existed an agent in $S^{*}$ unassigned, $i \in S^{*} \backslash \operatorname{Dom}(\mu)$, from $z \in C(\gamma)$ we would have $z_{i}=p_{i}>\eta_{i}$, in contradiction with the nucleolus being in the core.

Further,

$$
z_{k} \geq \eta_{k} \quad \text { for all } k \in M^{\prime} \backslash \mu\left(S^{*}\right) \quad \text { and } \quad z_{j}<\eta_{j} \quad \text { for all } j \in \mu\left(S^{*}\right) .
$$

Indeed, if $j \in \mu\left(S^{*}\right)$, then $j=\mu(i)$ for a unique $i \in S^{*}$ and the core property yields $z_{i}+z_{j}=a_{i j}=\eta_{i}+\eta_{j}$ and so, $z_{j}<\eta_{j}$ for all $j \in \mu\left(S^{*}\right)$. Similarly, if $k \in M^{\prime} \backslash \mu\left(S^{*}\right)$, either $k$ is not matched by $\mu$ and then $z_{k}=\eta_{k}=q_{k}$ or $k=\mu(t)$ for some $t \in M \backslash S^{*}$. In this second case, by (15), $z_{t} \leq \eta_{t}$, and since both $z$ and $\eta$ belong to the core, from $z_{t}+z_{k}=\eta_{t}+\eta_{k}=a_{t k}$ we get $z_{k} \geq \eta_{k}$.

Recall that (14) is valid for the nucleolus $\eta$ too since the nucleolus possesses both properties as shown before. Due to the inequalities (15)-(16) and the equality (14) applied to $S^{*}$ and $\eta$ respectively, it holds

$$
\max _{\substack{i \in S^{*}, k \in M^{\prime} \backslash \mu\left(S^{*}\right)}}\left\{p_{i}-z_{i}, \quad a_{i k}-z_{i}-z_{k}\right\}<\max _{\substack{i \in S^{*}, k \in M^{\prime} \backslash \mu\left(S^{*}\right)}}\left\{p_{i}-\eta_{i}, \quad a_{i k}-\eta_{i}-\eta_{k}\right\}
$$




$$
=\max _{\substack{j \in \mu\left(S^{*}\right), k \in M \backslash S^{*}}}\left\{q_{j}-\eta_{j}, \quad a_{k j}-\eta_{k}-\eta_{j}\right\}<\max _{\substack{j \in \mu\left(S^{*}\right), k \in M \backslash S^{*}}}\left\{q_{j}-z_{j}, \quad a_{k j}-z_{k}-z_{j}\right\} .
$$

The chain (17)-(18) of inequalities with at least one strict inequality contradicts (14) applied to coalition $S^{*}$. Hence, $z=\eta$.

The axioms in Theorem 1 are clearly independent because the core satisfies consistency with respect to Owen's reduced game on the class of assignment games but not symmetry of maximum complaints of the two sides and Thompson's fair division point satisfies the second axiom but fails to satisfy the first one, since it generally differs from the nucleolus.

Besides the above axiomatization result, we can provide a geometric characterization of the nucleolus for square assignment markets. To this end, some notations are needed. For all $S \subseteq M$, let the incidence vector $e^{S} \in \mathbb{R}^{M}$ be defined by $\left(e^{S}\right)_{i}=1$ for all $i \in S$ and $\left(e^{S}\right)_{i}=0$ for all $i \in M \backslash S$. The vector $e^{T} \in \mathbb{R}^{M^{\prime}}$, for all $T \subseteq M^{\prime}$, is defined analogously.

Now, let $\gamma=\left(M, M^{\prime}, A, p, q\right)$ be an assignment market with $|M|=\left|M^{\prime}\right|$. For each $\emptyset \neq S \subseteq M, \emptyset \neq T \subseteq M^{\prime},|S|=|T|$, we define the largest equal amount that can be transferred from players in $S$ to players in $T$ with respect to the core allocation $(u, v) \in C(\gamma)$, while remaining in the core of $\gamma$, by

$$
\delta_{S, T}^{\gamma}(u, v)=\max \left\{\varepsilon \geq 0 \mid\left(u-\varepsilon e^{S}, v+\varepsilon e^{T}\right) \in C(\gamma)\right\} .
$$

Similarly,

$$
\delta_{T, S}^{\gamma}(u, v)=\max \left\{\varepsilon \geq 0 \mid\left(u+\varepsilon e^{S}, v-\varepsilon e^{T}\right) \in C(\gamma)\right\} .
$$

We focus on the case in which $S$ and $T$ correspond one another by any optimal matching $\mu$, since whenever $\mu(S) \neq T$ the above critical amounts are null. Notice also that when a core element $(u, v) \in C(\gamma)$ satisfies $\delta_{S, \mu(S)}^{\gamma}(u, v)=\delta_{\mu(S), S}^{\gamma}(u, v)$, for some $\emptyset \neq S \subseteq M$, then this point $(u, v)$ bisects a certain core segment.

The following geometric characterization extends the bisection property provided by Maschler et al. (1979) to characterize the intersection of the kernel ${ }^{7}$ and the core of arbitrary coalitional games. In the case of Shapley and Shubik assignment games, it turns out that the kernel is always included in the core (Driessen, 1998) and a core element of $\gamma=\left(M, M^{\prime}, A\right)$ belongs to its kernel if and only if $\delta_{\{i\},\{\mu(i)\}}^{\gamma}(u, v)=$ $\delta_{\{\mu(i)\},\{i\}}^{\gamma}(u, v)$ for all $i \in M$ assigned by an optimal matching $\mu$ of $\gamma$.

Next theorem shows that, for square assignment games, if we require this bisection property to hold not only for all optimally matched pairs but for all optimally matched coalitions we geometrically characterize the nucleolus. This result generalizes the one given in Llerena and Núñez (2011) for the classical assignment game of Shapley and Shubik (1972).

Theorem 2 Let $\gamma=\left(M, M^{\prime}, A, p, q\right)$ be a square assignment market and $\mu \in \mathscr{M}_{\gamma}^{*}\left(M, M^{\prime}\right)$. Then, the nucleolus is the unique core allocation satisfying $\delta_{S, \mu(S)}^{\gamma}(\eta(\gamma))=\delta_{\mu(S), S}^{\gamma}(\eta(\gamma))$, for all $\emptyset \neq S \subseteq M$ with $|S|=|\mu(S)|$.

\footnotetext{
7 The kernel is a set-solution concept for coalitional games that was introduced by Davis and Maschler (1965). The kernel always contains the nucleolus.
} 
Proof Let $\gamma=\left(M, M^{\prime}, A, p, q\right)$ be a square assignment market, $\mu \in \mathscr{M}_{\gamma}^{*}\left(M, M^{\prime}\right)$. If $\mu=\emptyset$, the nucleolus is the only core point and we are done. If $\mu \neq \emptyset$, take any $\emptyset \neq S \subseteq M$ with $|S|=|\mu(S)|$. Given $(u, v) \in C(\gamma)$, the vector $\left(u-\varepsilon e^{S}, v+\varepsilon e^{\mu(S)}\right)$ belongs to the core if $u_{i}-\varepsilon \geq p_{i}$ for all $i \in S$, as well as $u_{i}-\varepsilon+v_{j} \geq a_{i j}$ for all $i \in S$, $j \in M^{\prime} \backslash \mu(S)$. Equivalently,

$$
\varepsilon \leq u_{i}-p_{i} \quad \text { for all } i \in S \text { and } \quad \varepsilon \leq u_{i}+v_{j}-a_{i j} \quad \text { for all } i \in S, j \in M^{\prime} \backslash \mu(S) .
$$

Hence, by (19) and (20), the critical numbers $\delta_{S, \mu(S)}^{\gamma}(u, v)$ and $\delta_{\mu(S), S}^{\gamma}(u, v)$ equal

$$
\begin{aligned}
& \delta_{S, \mu(S)}^{\gamma}(u, v)=-\max _{\substack{i \in S, k \in M^{\prime} \backslash \mu(S)}}\left\{p_{i}-u_{i}, \quad a_{i k}-u_{i}-v_{k}\right\}, \\
& \delta_{\mu(S), S}^{\gamma}(u, v)=-\max _{\substack{j \in \mu(S), k \in M \backslash S}}\left\{q_{j}-v_{j}, \quad a_{k j}-u_{k}-v_{j}\right\} .
\end{aligned}
$$

By the same argument as in the proof of Theorem 1, the equality $\delta_{S, \mu(S)}^{\gamma}(u, v)=$ $\delta_{\mu(S), S}^{\gamma}(u, v)$ occurs if and only if $(u, v)=\eta(\gamma)$, being $\eta(\gamma)$ the nucleolus of $\gamma$.

Notice from equations (21) and (22) that, when applied to a solution point $(u, v) \in$ $\sigma(\gamma)$ that belongs to the core, the symmetry of maximum complaints of the two sides, $\max _{i \in M}\left\{p_{i}-u_{i}\right\}=\max _{j \in M^{\prime}}\left\{q_{j}-v_{j}\right\}$, coincides with the bisection condition $\delta_{M, M^{\prime}}^{\gamma}(u, v)=\delta_{M^{\prime}, M}^{\gamma}(u, v)$. This means that core elements of square assignment markets satisfying symmetry of maximum complaints of the two sides, are at the midpoint of a certain $45^{\circ}$-slope range within the core. The above theorem shows that, in addition to this one, the nucleolus satisfies other bisection conditions.

Figure 2 illustrates the above geometric characterization of the nucleolus.

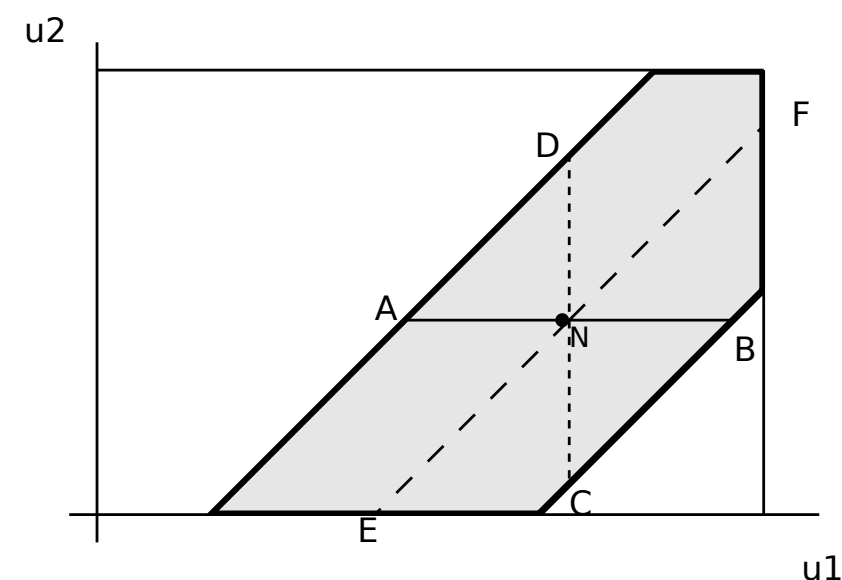

Fig. 2 
In light grey we represent the core of a $2 \times 2$ assignment game in the plane $u_{1}, u_{2}$ of the buyers' payoffs. If we assume that $\mu=\{(1,1),(2,2)\}$ is an optimal matching, then the nucleolus is the unique core allocation (denoted by $N$ in the picture) that bisects at the same time the horizontal segment $[A, B]$, the vertical segment $[C, D]$ and the $45^{\circ}$-slope segment $[E, F]$. The higher the dimension of the core, the more the number of bisection equalities that must be considered.

We finish with two concluding remarks. The above characterization of the nucleolus of square assignment markets applies to arbitrary assignment markets only adding dummy agents on the short side, that is agents with null reservation values and null row or column in the assignment matrix. Secondly, this geometric characterization has been used by Martínez de Albéniz et al. (2013a, 2013b) to provide a procedure for the computation of the nucleolus of assignment games.

\section{References}

1. van den Brink R, Pintér M (2012) On axiomatizations of the Shapley value for assignment games, Tinbergen Institute Discussion Paper n. 12-092/II

2. Davis M, Maschler M (1965) The kernel of a cooperative game. Naval Research Logistics Quarterly 12: 223-259

3. Driessen TSH (1991) A survey of consistency properties in cooperative game theory, SIAM Review 33: $43-59$

4. Driessen TSH (1998) A note on the inclusion of the kernel in the core of the bilateral assignment game. International Journal of Game Theory 27: 301-303

5. Driessen TSH (1999) Pairwise-bargained consistency and game theory: the case of a two-sided firm Fields Institute Communications Series 23: 65-82

6. Hoffmann M, Sudhölter P (2007) The Shapley value of exact assignment games. International Journal of Game Theory 35: 557-568

7. Llerena F, Núñez M (2011) A geometric characterization of the nucleolus of the assignment game Economics Bulletin 31: 3275-3285

8. Martínez de Albéniz FJ, Rafels C and Ybern N (2013a) On the nucleolus of $2 \times 2$ assignment games Economics Bulletin 2: 1641-1648

9. Martínez de Albéniz FJ, Rafels C and Ybern N (2013b) A procedure to compute the nucleolus of the assignment game. Operations Research Letters, 41, 675-678

10. Maschler M, Peleg B, Shapley S (1979) Geometric properties of the kernel, nucleolus and related solution concepts. Mathematics of Operations Research 4: 303-338

11. Miquel S, Núñez M (2011) The maximum and the addition of assignment games. TOP 19: 189-212

12. Núñez M, Rafels C (2013) Von Neumann-Morgenstern solutions to the assignment market. Journal of Economic Theory 148: 1282-1291

13. Owen G (1992) The assignment game: The reduced game. Annales d'Économie et de Statistique 25/26: 71-79

14. Peleg B (1986) On the reduced game property and its converse. International Journal of Game Theory 15: $187-200$

15. Peleg B and Sudhölter P (2007) Introduction to the theory of cooperative games. 2nd edition. Springer.

16. Potters J (1991) An axiomatization of the nucleolus. International Journal of Game Theory 19: 365373

17. Potters J, Tijs S (1992) The nucleolus of matrix games and other nucleoli. Mathematics of Operations Research 17: 164-174

18. Rochford SC (1984) Symmetrically pairwise-bargained allocations in an assignment market. Journal of Economic Theory 34: 262-281

19. Sasaki H (1995) Consistency and monotonicity in assignment problems. International Journal of Game Theory 24: 373-397

20. Shapley LS, Shubik M (1972) The Assignment Game I: The Core. International Journal of Game Theory 1: 111-130 
21. Schmeidler D (1969) The nucleolus of a characteristic function game. SIAM Journal of Applied Mathematics 17: 1163-1170

22. Sobolev AI (1975) The characterization of optimality principles in cooperative games by functional equations. In: Vorobiev NN (ed) Math Methods Social Sci 6, Academy of Sciences of the Lithunian SSR, Vilnius 95-151 (in Russian)

23. Solymosi T, Raghavan TES (1994) An algorithm for finding the nucleolus of assignment games. International Journal of Game Theory 23: 119-143

24. Toda M (2003) Consistency and its converse in assignment games. International Journal of Mathematics, Game Theory and Algebra 13: 1-14

25. Toda M (2005) Axiomatization of the core of assignment games. Games and Economic Behavior 53: 248-261

26. Thomson W (2003) Consistent allocation rules. Mimeo. University of Rochester

27. Thompson GL (1981) Auctions and market games. In: Essays in Game Theory and Mathematical Economics in honour of Oskar Morgenstern. Bibliographisches Institute AG, Zurich, 181-196 